\title{
IFIT Proteins Are Involved in CXCL10 Expression in Human Glomerular Endothelial Cells Treated with a Toll-Like Receptor 3 Agonist
}

\author{
Tadaatsu Imaizumi $^{a}$ Shun Hashimoto ${ }^{b}$ Riko Sato $^{b}$ Hidenori Umetsu $^{b}$

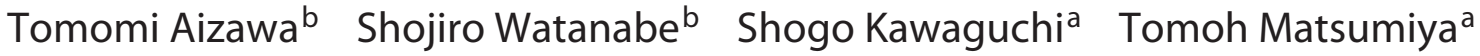 \\ Kazuhiko Seya ${ }^{a}$ Jiangli Ding ${ }^{a}$ Hiroshi Tanakab, c $^{\text {Ta }}$ \\ aDepartment of Vascular Biology, Institute of Brain Science, Hirosaki University Graduate School of Medicine, \\ Hirosaki, Japan; ${ }^{b}$ Department of Pediatrics, Hirosaki University Graduate School of Medicine, Hirosaki, Japan; \\ 'Department of School Health Science, Hirosaki University Faculty of Education, Hirosaki, Japan
}

\section{Keywords}

CXCL10 · IFIT1 · IFIT2 $\cdot$ IFIT3 $\cdot$ Glomerular endothelial cells

\begin{abstract}
Introduction: Various viruses including a novel coronavirus (SARS-CoV-2) can infect the kidney. When viruses invade the glomeruli from the bloodstream, glomerular endothelial cells (GECs) initiate the innate immune reactions. We investigated the expression of interferon (IFN)-induced protein with tetratricopeptide repeats (IFIT) $1 / 2 / 3$, antiviral molecules, in human GECs treated with a toll-like receptor (TLR) 3 agonist. Role of IFIT1/2/3 in the expression of C-X-C motif chemokine ligand 10 (CXCL10) was also examined. Methods: Human GECs were cultured and stimulated with polyinosinic-polycytidylic acid (poly IC), a synthetic TLR3 agonist. Realtime $\mathrm{qPCR}$, Western blotting, and ELISA were used to examine the expression of IFIT1/2/3, IFN- $\beta$, and CXCL10. RNA interference against IFN- $\beta$ or IFIT1/2/3 was also performed. Results: Expression of IFIT1/2/3 and CXCL10 was induced by poly IC in GECs. The inductions were inhibited by RNA interfering of IFN- $\beta$. Knockdown of IFIT1/2/3 decreased the CXCL10 expression. Knockdown of IFIT3 decreased the expression of IFIT1 and IFIT2 proteins. Conclusion: IFIT1/2/3
\end{abstract}

karger@karger.com www.karger.com/kbr

Karger"

BOPEN ACCESS
(C) 2020 The Author(s)

Published by S. Karger AG, Basel

This article is licensed under the Creative Commons AttributionNonCommercial-NoDerivatives 4.0 International License (CC BYNC-ND) (http://www.karger.com/Services/OpenAccessLicense). Usage and distribution for commercial purposes as well as any distribution of modified material requires written permission. and CXCL10 were induced by poly IC via IFN- $\beta$ in GECs. IFIT1/2/3 may increase the expression of CXCL10 which induces lymphocyte chemotaxis and may inhibit the replication of infected viruses. These molecules may play a role in GEC innate immune reactions in response to viruses.

(c) 2020 The Author(s).

Published by S. Karger AG, Basel

\section{Introduction}

Viral infections are common triggers of onset or worsening of glomerular nephritis [1]. Several mechanisms are likely involved in the pathogenesis of virus-related nephropathy. Deposition of antigen-antibody complexes in glomeruli is a recognized pathogenic mechanism for glomerulonephritis. Recently, the innate immune system was also demonstrated to contribute to the activity of glomerulonephritis in diseases such as lupus nephritis [2]. Binding of the non-self-pathogen-associated molecular patterns to pattern recognition receptors (PRRs) initiates innate immune reactions. The self-damage-associated molecular patterns released by damaged or injured cells can be also recognized by PRRs, leading to non-infectious "sterile inflammation" [3]. 
Toll-like receptor (TLR) 3 is one of the PRRs [4]. TLR3 can recognize viral double-stranded RNA (dsRNA), which is a replication intermediate for many viruses. Binding of viral dsRNA to TLR3 initiates antiviral innate immune reactions. TLR3 can also recognize some forms of endogenous RNA released from necrotic cells [5], followed by activation of pseudoviral innate immune reactions [6]. Binding of dsRNA ligand to TLR3 activates a downstream signaling cascade and induces the expression of various molecules such as type I interferons (IFNs) and other cytokines. Type I IFNs are principal cytokines in antiviral innate immune reactions. The broad functions of type I IFNs involve the expression of hundreds of IFN-stimulated genes (ISGs). Activation of TLR3/type I IFNs/ISGs axis by viral dsRNA may be important in antiviral host defense and in the onset or worsening of glomerular diseases such as lupus nephritis [6] and IgA nephropathy [7]. In addition, pseudoviral immune responses induced by self-damage-associated molecular patterns may also activate TLR3/ type I IFNs/ISGs axis in glomerulonephritis even in the absence of obvious viral infection [8].

The glomerulus filters blood to form primary urinary filtrate. When viruses reach the glomeruli from the circulation, the first cells they contact are glomerular endothelial cells (GECs). Therefore, GECs may play an important role in defense against viral infections in the kidney. We previously observed that TLR3 is expressed in human GECs [9]. A synthetic dsRNA polyinosinic-polycytidylic acid (poly IC) is a ligand of TLR3. Poly IC is widely used as a mimic of viral or endogenous dsRNA [10]. Treatment of GECs with poly IC can induce IFN- $\beta$ expression via TLR3. The newly synthesized IFN- $\beta$ is involved in the expression of E-selectin, C-X-C motif chemokine ligand 1 (CXCL1) [9], retinoic acid-inducible gene-I, melanoma differentiation-associated gene 5, interleukin-6 [11], and CX3CL1 [12]. However, the antiviral and pseudoviral responses downstream of TLR3/IFN- $\beta$ activation have not been fully elucidated in human GECs.

IFN-induced protein with tetratricopeptide repeats 1 (IFIT1; also termed as ISG56), IFIT2 (also termed as ISG54), and IFIT3 (also termed as ISG60) are ISGs. They encode proteins with multiple IFIT motifs [13]. IFIT proteins lack enzymatic domains but have a wide range of biological functions by mediating protein-protein and protein-RNA interactions [14]. The IFIT proteins are involved in many processes in response to viral infection [15]. The interactions of IFIT proteins inhibit viral infections through multiple mechanisms, including translation inhibition, dsRNA signaling, cells proliferation, cell death, and sequestering viral proteins or RNA in the cytoplasm [13]. It has been re-

IFIT1, IFIT2, and IFIT3 Regulate CXCL10

in Glomerular Endothelial Cells ported that IFIT1 inhibits translation of non-self-RNA by binding directly [16] and IFIT2 plays a critical role in defense of viral infections by promoting apoptosis of infected cells [17]. Dengue virus infection-induced expression of IFIT proteins and knockdown of IFIT3 resulted in an increase of dengue virus infectivity [18]. Although IFIT proteins are thought to be important antiviral proteins, there have been no reports on the expression and function of IFIT proteins in GECs. In the present study, we explored the hypothesis that IFIT1, IFIT2, and IFIT3 are expressed and play a role in antiviral reactions in cultured human GECs treated with poly IC.

CXCL10 is a member of CXC chemokine family [19]. CXC motif chemokine receptor 3 (CXCR3) is the receptor for CXCL10 [20]. The expression of CXCL10 can be upregulated by IFNs. CXCL10 induces the chemotaxis of lymphocytes expressing CXCR3 and is crucial in the pathogenesis of glomerulonephritis [21]. We also presently investigated the role of IFIT proteins in the expression of CXCL10 in GECs.

\section{Materials and Methods}

\section{Reagents}

EGM-2 endothelial growth medium was purchased from Lonza (Walkersville, MD, USA). Poly IC and an anti-actin rabbit IgG (A5060) were from Sigma (St. Louis, MO, USA). Recombinant human $(\mathrm{r}[\mathrm{h}])$ IFN- $\beta$ was from ProSpec (East Brunswick, NJ, USA). Lipofectamine RNAiMAX was from Invitrogen (Frederick, MD, USA). A non-silencing negative control siRNA (1027281) and siRNA against IFIT1 (SI00445879), IFIT2 (SI04145372), or IFIT3 (SI04197788) were from Qiagen (Hilden, Germany). IFN- $\beta$ siRNA was described previously [22]. The Illustra RNA spin kit was from GE Healthcare (Buckinghamshire, UK). Moloney Murine Leukemia Virus reverse transcriptase was from Thermo Fisher Scientific (Asheville, MA, USA). Oligo $(\mathrm{dT})_{18}$ primer and primers for PCR were custom synthesized by Greiner Japan (Atsugi, Japan). The Sso-Advanced Universal SYBR Green Supermix was from Bio-Rad (Hercules, CA, USA). Horseradish peroxidase (HRP)-labeled anti-rabbit IgG antibody was from Medical \& Biological Laboratories (Nagoya, Japan). PVDF membrane and Immobilon Crescendo Western HRP substrate were from Merck Millipore (Temecula, CA, USA). Rabbit polyclonal antibodies against IFIT1 (GTX118713), IFIT2 (GTX108346), or IFIT3 (GTX112442) were from GeneTex (Irvine, CA, USA). ELISA kits for CXCL10 and IFN- $\beta$ were purchased from R\&D Systems (Minneapolis, MN, USA) and PBL Assay Science (Piscataway, NJ, USA), respectively.

\section{Cell Culture}

Primary human GECs purchased from ScienCell (San Diego, CA, USA) were cultured in EGM-2 medium. The culture dishes and plates were pre-coated with human gelatin. The cells were treated with $0.1-50 \mu \mathrm{g} / \mathrm{mL}$ poly IC for up to $24 \mathrm{~h}$. RNA interference against IFN- $\beta$, IFIT1, IFIT2, or IFIT3 was performed as follows. 
When the cells were approximately $40 \%$ confluent, the cells were transfected with an siRNA complexed with Lipofectamine RNAiMAX reagent according to the manufacturer's protocol. After 48-h incubation, the medium was changed and the cells were treated with $30 \mu \mathrm{g} / \mathrm{mL}$ poly IC. After incubation for indicated times, RNA and proteins were extracted from the cells and the conditioned medium was collected. The expression of mRNA was estimated using real-time qRT-PCR. The proteins in cell lysates or in the conditioned medium were analyzed using Western blotting or ELISA, respectively.

Quantitative real-time reverse transcription-PCRTo examine the expression of mRNAs in cells, total RNA was extracted from the cells using the illustra RNA spin kit. RNA was purified and reverse-transcribed into cDNA using oligo $(\mathrm{dT})_{18}$ and Moloney Murine Leukemia Virus reverse transcriptase. The cDNAs for IFIT1, IFIT2, IFIT3, CXCL10, IFN- $\beta$, and glyceraldehyde-3-phosphate dehydrogenase (GAPDH) were amplified using specific primers and Sso-Advanced Universal SYBR Green Supermix. CFX96 real-time PCR detection system and CFX Manager ${ }^{\mathrm{TM}}$ software (Bio-Rad) were used. A standard curve was made in every PCR, and specificity of the PCR was confirmed by sequencing the products. The results for IFIT1, IFIT2, IFIT3, and IFN- $\beta$ mRNAs were shown as "fold increase" compared to those for cells that were not treated with poly IC. CXCL10 cDNA was not detected in cells without poly IC treatment, and "arbitrary units" was used for the data. Primers used were as follows:

IFIT1-F: 5'-TAGCCAACATGTCCTCACAGAC-3', IFIT1-R: 5'-TCTTCTACCACTGGTTTCATGC-3', IFIT2-F: 5'-GGTCTCTTCAGCATTTATTGGTG-3', IFIT2-R: 5' -TGCCGTAGGCTGCTCTCCA-3', IFIT3-F: 5'-GAACATGCTGACCAAGCAGA-3', IFIT3-R: 5' -CAGTTGTGTCCACCCTTCCT-3', CXCL10F: $\quad 5^{\prime}$-TTCAAGGAGTACCTCTCTCTAG-3', CXCL10-R: 5'-CTGGATTCAGACATCTCTTCTC-3', CXCL1-F: 5'-ATGGCCCGCGCTGCTCTCTCC-3', CXCL1-R: 5'-GTTGGATTTGTCACTGTTCAG-3', IFN- $\beta$-F: $5^{\prime}$-CCTGTGGCAATTGAATGGGAGGC-3', IFN- $\beta$-R: $\quad 5^{\prime}$-CCAGGCACAGTGACTGTACTCCTT-3', GAPDH-F: 5'-GCACCGTCAAGGCTGAGAAC- $3^{\prime}$, and GAPDH-R: 5' -ATGGTGGTGAAGACGCCAGT- $3^{\prime}$.

\section{Western Blotting}

The cells were washed twice with PBS after the incubation and were lysed using Laemmli reducing sample buffer. The lysates were subjected to polyacrylamide (5-20\%) gel electrophoresis. The proteins were transferred to a PVDF membrane, and the membrane was probed with an antibody against IFIT1 (1:3,000 dilution), IFIT2 (1:500), IFIT3 $(1: 5,000)$, or actin $(1: 5,000)$. The bands were detected using a HRP-labeled anti-rabbit IgG antibody and Immobilon Crescendo Western HRP chemiluminescence substrate.

\section{Enzyme-Linked Immunosorbent Assay}

CXCL10 and IFN- $\beta$ proteins in the cell-conditioned medium were measured using ELISA kits according to the manufacturer's instruction using a Bio-Rad iMark ${ }^{\mathrm{TM}}$ plate reader.

\section{Statistics}

Data for qRT-PCR and ELISA were presented in terms of means \pm SD of biological triplicate experiments. Statistical analysis for qRT-PCR and ELISA was performed using unpaired 2-tailed $t$ test $(n=3)$.

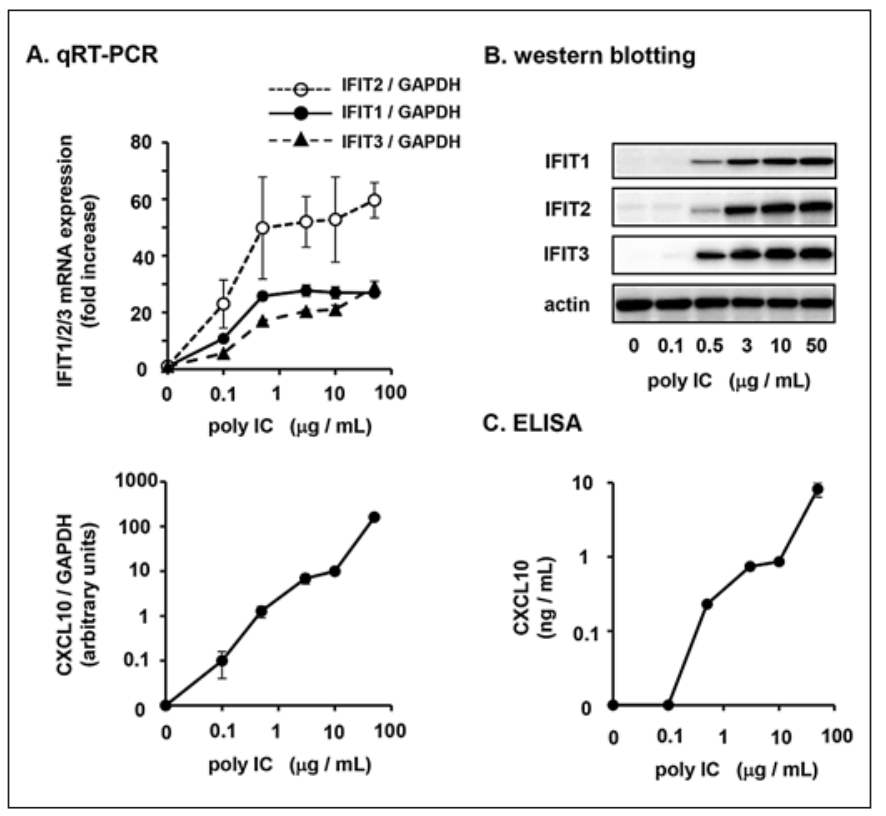

Fig. 1. Poly IC induces the expression of IFIT1, IFIT2, IFIT3, and CXCL10 in cultured human GECs in a concentration-dependent manner. A Poly IC was added to the culture at the concentration of $0.1-50 \mu \mathrm{g} / \mathrm{mL}$ poly IC, and the cells were incubated for $16 \mathrm{~h}$. The expression of mRNA for IFIT1, IFIT2, IFIT3, CXCL10, and GAPDH was estimated using qRT-PCR analysis. Briefly, RNA was extracted from the cells, and cDNA was synthesized from the purified RNA. Each cDNA was amplified with specific primers using real-time PCR system. The expression of each mRNA was normalized to the value of GAPDH. The results for IFIT1, IFIT2, IFIT3, and IFN- $\beta$ mRNAs were shown as "fold increase" compared to those for cells that were not treated with poly IC (upper panel). CXCL10 cDNA was not detected in cells without poly IC treatment, and "arbitrary units" was used for the data (lower panel). B After treating with $0.1-50 \mu \mathrm{g} / \mathrm{mL}$ poly IC for $24 \mathrm{~h}$, the cells were lysed using Laemmli sample buffer. The expression of IFIT1, IFIT2, IFIT3, and actin proteins was examined using Western blotting. C The cells were treated with poly IC as in (A). After $24-\mathrm{h}$ incubation, the medium was collected from the culture. CXCL10 concentration in the medium was measured using an ELISA kit. The results in $(\mathbf{A})$ and $(\mathbf{C})$ are shown as mean \pm SD of triplicate experiments. Poly IC, polyinosinic-polycytidylic acid; GECs, glomerular endothelial cells; IFIT1/2/3, tetratricopeptide repeats 1/2/3; CXCL, C-X-C motif chemokine ligand; GAPDH, glyceraldehyde-3-phosphate dehydrogenase; IFN, interferon.

\section{Results}

Normal human GECs cultured without stimulation displayed small amounts of IFIT1, IFIT2, and IFIT3 mRNA. CXCL10 mRNA was not detected. When the cells were treated with poly IC, the expression levels of IFIT1, IFIT2, IFIT3, and CXCL10 mRNA increased in a concentration-dependent manner (Fig. 1A). The in- 


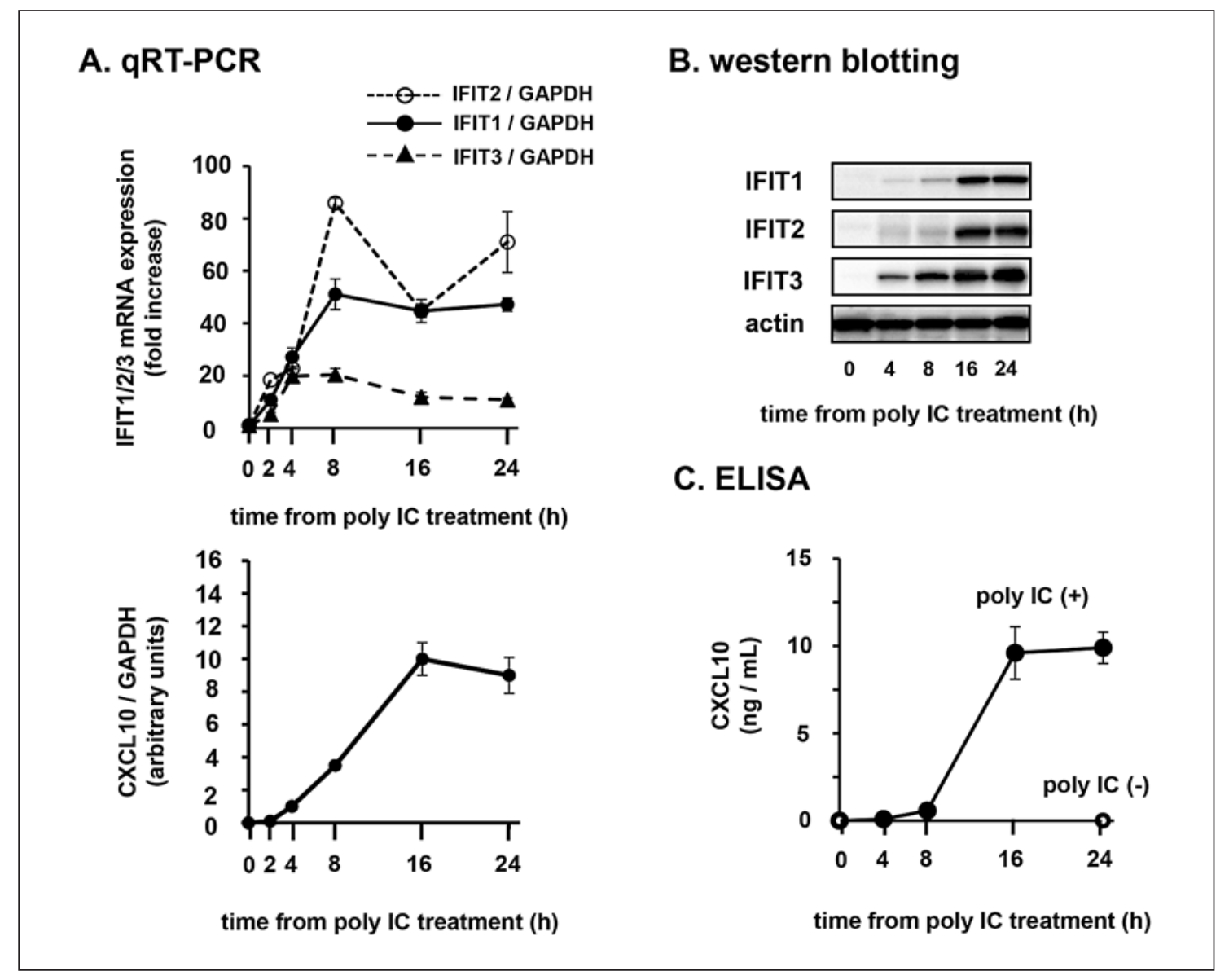

Fig. 2. Poly IC induces the expression of IFIT1, IFIT2, IFIT3, and CXCL10 in human GECs in a time-dependent manner. The cells were treated with poly IC with $30 \mu \mathrm{g} / \mathrm{mL}$ poly IC for up to $24 \mathrm{~h}$, and the expression of mRNA and protein for IFIT1, IFIT2, IFIT3, and CXCL10 was analyzed using qRT-PCR (A), Western blotting (B), and ELISA $(\mathbf{C})$ as in Fig. 1. The results in $(\mathbf{A})$ and $(\mathbf{C})$ are shown as mean \pm SD of triplicate experiments. Poly IC, polyinosinic-polycytidylic acid; GECs, glomerular endothelial cells; IFIT1/2/3, tetratricopeptide repeats 1/2/3; CXCL, C-X-C motif chemokine ligand; GAPDH, glyceraldehyde-3-phosphate dehydrogenase.

crease of mRNA for IFIT1 and IFIT2 nearly plateaued in cells treated with $>0.5 \mu \mathrm{g} / \mathrm{mL}$, while IFIT3 mRNA increased gradually in cells treated with $0.1-50 \mu \mathrm{g} / \mathrm{mL}$ poly IC. CXCL10 mRNA expression was gradually induced by $0.5-10 \mu \mathrm{g} / \mathrm{mL}$ poly IC, and the induction was dramatically increased by $50 \mu \mathrm{g} / \mathrm{mL}$ poly IC. The protein expression of IFIT1, IFIT2, IFIT3 (Fig. 1B), and CXCL10 (Fig. 1C) was also upregulated by poly IC. Increase in protein expression of these molecules almost paralleled mRNA expression. However, IFIT1 and IFIT 2 protein expression induced by $0.5 \mu \mathrm{g} / \mathrm{mL}$ poly IC seemed to be weak when compared with the expression of those mRNAs. Some posttranscriptional regulation may exist in the expression of IFIT1 and IFIT2.
Poly IC also induced the expression of IFIT1, IFIT2, IFIT3, and CXCL10 in a time-dependent manner. The mRNA expression of IFIT1, IFIT2, and IFIT3 began to increase quickly after the poly IC treatment, and maximal levels of these ISGs were observed at $8 \mathrm{~h}$. The increase of CXCL10 mRNAs was relatively slower. CXCL10 mRNA expression reached its maximum at $16 \mathrm{~h}$ after the stimulation. The protein expression of IFIT1, IFIT2, and IFIT3 lagged behind the corresponding mRNA expression. The maximal levels of these proteins were observed at 16-24 h (Fig. 2B). Time course of CXCL10 protein expression almost paralleled that of mRNA expression (Fig. 2C).

Transfection of cells with siRNA against IFN- $\beta$ significantly reduced the mRNA expression of IFIT1, IFIT2, 
Fig. 3. IFN- $\beta$ is involved in the poly IC-induced expression of IFIT1, IFIT2, IFIT3, and CXCL10 in human GECs. A-C The cells were transfected with a non-silencing negative control siRNA or a specific siRNA against IFN- $\beta$. After incubating for $48 \mathrm{~h}$, the medium was changed and the cells were treated with $30 \mu \mathrm{g} / \mathrm{mL}$ poly IC. A The cells were subjected to qRT-PCR analysis for IFIT1, IFIT2, IFIT3, and CXCL10 mRNA $16 \mathrm{~h}$ after poly IC treatment, as in Fig. 1. IFN- $\beta$ mRNA expression was examined as well $2 \mathrm{~h}$ after poly IC treatment $(n=3)$. B The cells were lysed $24 \mathrm{~h}$ after poly IC treatment, and Western blotting for IFIT1, IFIT2, IFIT3, and actin was performed. C The cell-conditioned medium was collected $24 \mathrm{~h}$ after poly IC treatment, and the concentration of CXCL10 and IFN- $\beta$ was examined using ELISA $(n=$ 3). D-F The cells were treated with $1 \mathrm{ng} / \mathrm{mL}$ r(h)IFN- $\beta$ for 16 h. D RNA was extracted from the cells and qRT-PCR analysis for IFIT1, IFIT2, and IFIT3 (upper panel) and CXCL10 (lower panel) was performed ( $n=$ $3)$. E The cells were lysed, and the expression of IFIT1, IFIT2, IFIT3, and actin proteins was examined using Western blotting. F The concentration of CXCL10 protein in the conditioned medium was analyzed by an ELISA $(n=3)$. Poly IC, polyinosinic-polycytidylic acid; GECs, glomerular endothelial cells; IFIT1/2/3, tetratricopeptide repeats $1 / 2 / 3$; CXCL, C$\mathrm{X}-\mathrm{C}$ motif chemokine ligand; GAPDH, glyceraldehyde-3-phosphate dehydrogenase; IFN, interferon; $\mathrm{r}(\mathrm{h}) \mathrm{IFN}$, recombinant human IFN.

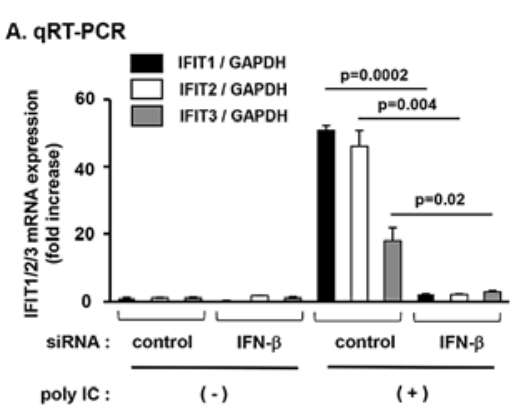

B. western blotting

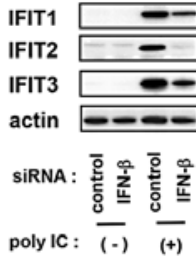

C. ELISA
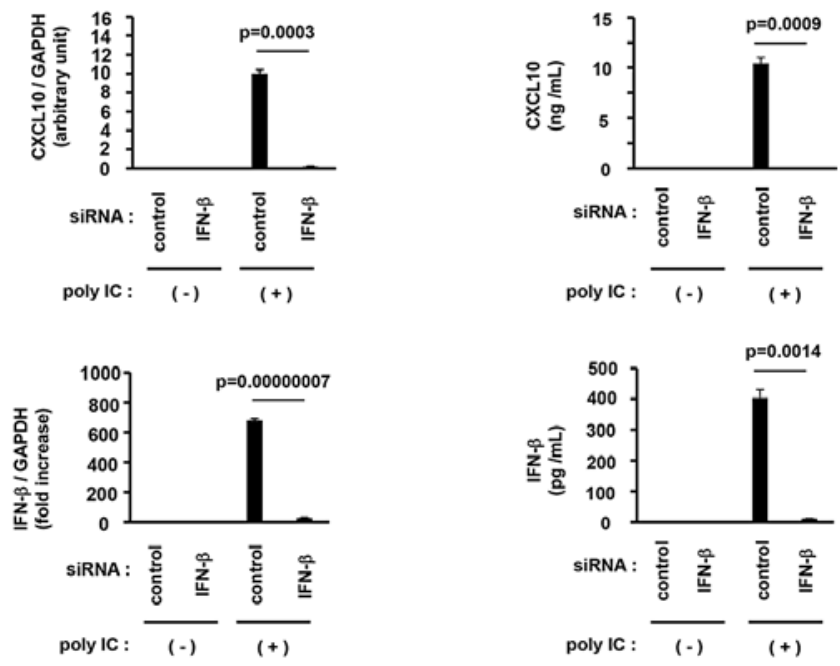

\section{D. $\mathrm{qRT}-\mathrm{PCR}$}

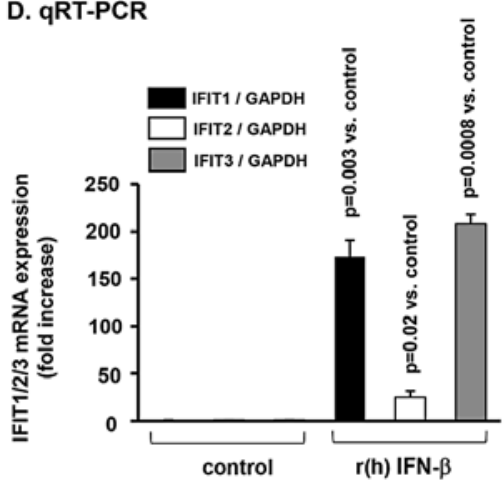

E. western blotting

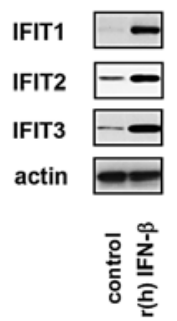

F. ELISA

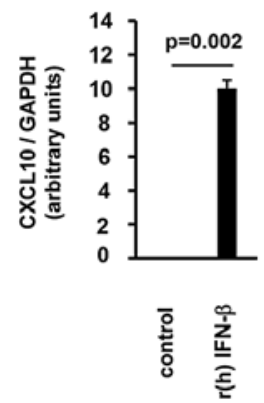


IFIT3, and CXCL10 induced by poly IC (Fig. 3A). Effective knockdown of IFN- $\beta$ was confirmed (Fig. $3 \mathrm{~A}, \mathrm{C}$ ). The knockdown of IFN- $\beta$ also decreased the poly IC-induced expression of proteins for IFIT1, IFIT2, IFIT3 (Fig. 3B), and CXCL10 (Fig. 3C). In addition, treatment of cells with $\mathrm{r}(\mathrm{h})$ IFN- $\beta$ induced mRNA (Fig. 3D) and protein expression of IFIT1, IFIT2, IFIT3 (Fig. 3E), and CXCL10 (Fig. 3F).

Transfection of cells with siRNA against IFIT1, IFIT2, or IFIT3 resulted in the decrease of CXCL10 mRNA (Fig. 4A) and protein (Fig. 4B) expression induced by poly IC. Knockdown of IFIT1, IFIT2, or IFIT3 did not affect poly IC-induced mRNA expression of CXCL1 and IFN- $\beta$ (Fig. 4A). Knockdown of IFIT1, IFIT2, and IFIT3 by RNA interference was confirmed using Western blotting (Fig. 4C). RNA interference of IFIT3 also resulted in inhibition of the expression of IFIT1 and IFIT2 proteins. Knockdown of IFIT1 decreased the expression of IFIT2 protein. However, IFIT3 protein expression was not affected by knockdown of IFIT1 or IFIT2 (Fig. 4C).

\section{Discussion}

The novelty of the present study is that IFIT1, IFIT2, IFIT3, and CXCL10 were markedly induced by poly IC in cultured human GECs. These observations suggest that IFIT1, IFIT2, IFIT3, and CXCL10 may be involved in antiviral and pseudoviral innate immune reactions in human GECs. Since all of these molecules are encoded by ISGs, RNA interference of IFN- $\beta$ was performed. Knockdown of IFN- $\beta$ decreased the poly IC-induced upregulation of all of IFIT1, IFIT2, IFIT3, and CXCL10. In addition, treatment of cells with $\mathrm{r}(\mathrm{h}) \mathrm{IFN}-\beta$ induced the expression of these molecules. These results indicate that newly synthesized IFN- $\beta$ mediates poly IC-induced expression of IFIT1, IFIT2, IFIT3, and CXCL10 in an autocrine and/or paracrine manner.

IFIT proteins exert antiviral functions via multiple mechanisms [13]. For example, IFIT proteins can inhibit viral infection by binding directly to non-self-RNAs to inhibit their translation or replication [23]. IFIT1 inhibits translation by binding directly to $2^{\prime} \mathrm{O}$-unmethylated capped RNA $[16,24]$. IFIT2 modulates the stability of cytokine mRNA by binding directly to RNA with adenylateuridylate-rich RNAs [25]. In the present study, the mRNA levels for IFIT1, IFIT2, and IFIT3 were greatest $8 \mathrm{~h}$ after poly IC treatment. The increase of CXCL10 mRNA was slower than those of IFIT1, IFIT2, and IFIT3 and reached maximum $16 \mathrm{~h}$ after the poly IC treatment. It has been reported that overexpression of IFIT1 upregulated CXCL10 expression in B cells [26]. In the present study, knockdown of IFIT1, IFIT2, or IFIT3 resulted in the decrease of CXCL10 expression induced by poly IC, suggesting that IFIT1, IFIT2, and IFIT3 positively regulate the expression of CXCL10 in GECs. Increased expression of CXCL10 may lead to lymphocyte recruitment, followed by activation of adaptive immune reactions. Therefore, positive regulation of CXCL10 may be a new function of IFIT proteins in antiviral reactions in GECs. These results support our previous study in U373MG astrocytoma cells [27, 28]. However, the results of the present study may contradict the previous observations that poly IC-induced CXCL10 expression is negatively regulated by IFIT3 in hCMEC/D3 brain microvascular endothelial cells [29] and that IFIT1 is a mediator of negative-feedback regulation of virus-triggered antiviral response in 293 cells [30]. It is conceivable that IFIT proteins may differentially interact with multiple molecules in different pathophysiological conditions and may exert cell typespecific functions.

The TLR3/IFN- $\beta$ /IFITs/CXCL10 axis may contribute to antiviral defense in GECs. The involvement of TLR3 [31], IFN- $\beta$ [32], and CXCL10 [33] in the pathogenesis of inflammatory renal diseases has been demonstrated. Therefore, dysregulation or excessive activation of the TLR3/IFN- $\beta$ /IFITs/CXCL10 axis may play a role in the pathogenesis of glomerular nephritis.

For optimal IFIT function, protein-protein interactions are necessary. IFIT proteins can associate with each other to form functional heterocomplexes, and these interactions can modulate the functions of different IFITs. IFIT2 and IFIT3 stabilize IFIT1 protein expression and enhance translation inhibition activity of IFIT1 in HEK293T cells [34]. When we observed the time course of the expression of IFIT1, IFIT2, and IFIT3 proteins in poly IC-stimulated GECs, the expression of IFIT3 protein began earlier than that of IFIT1 and IFIT2. In addition, knockdown of IFIT3 resulted in the decreased expression of IFIT1 and IFIT2 proteins, while IFIT3 protein expression was not affected by knockdown of IFIT1 or IFIT2. It has been reported that IFIT3 serves as a central scaffold to regulate the antiviral functions of IFIT1 and IFIT2 [34] and IFIT3 protein enhances the stability of IFIT1 protein [35]. In addition, knockdown of IFIT1 resulted in the decrease of IFIT2 protein. Taken together, we speculate that IFIT3 protein stabilizes IFIT1 and IFIT2 proteins and IFIT1 protein may stabilize IFIT2 protein. We did not confirm the direct proteinprotein binding among IFIT proteins in the current study. It should be clarified in further studies. 
Fig. 4. IFIT1, IFIT2, and IFIT3 positively regulate the expression of CXCL10, and IFIT3 mediates the expression of IFIT1 and IFIT2 proteins in human GECs treated with poly IC. A The cells were transfected with a non-silencing control siRNA or a specific siRNA against IFIT1, IFIT2, or IFIT3. After incubating for $48 \mathrm{~h}$, poly IC was added to the culture at the concentration of $30 \mu \mathrm{g} / \mathrm{mL}$ poly IC. A RNA was extracted from the cells $16 \mathrm{~h}$ after poly IC treatment and was subjected to qRT-PCR for CXCL10 (upper panel) and CXCL1 (middle panel). The cells were also subjected to qRT-PCR for IFN- $\beta 2 \mathrm{~h}$ after poly IC treatment (lower panel) $(n=3)$. B The cellconditioned medium was collected $24 \mathrm{~h}$ after poly IC treatment, and CXCL10 protein concentration in the medium was measured with ELISA $(n=3)$. C The cells were lysed $24 \mathrm{~h}$ after poly IC treatment, and Western blotting for IFIT1, IFIT2, and IFIT3 proteins was performed. Poly IC, polyinosinic-polycytidylic acid; GECs, glomerular endothelial cells; IFIT1/2/3, tetratricopeptide repeats $1 / 2 / 3$; CXCL, C-X-C motif chemokine ligand; GAPDH, glyceraldehyde-3-phosphate dehydrogenase; IFN, interferon.

\section{A. qRT-PCR}
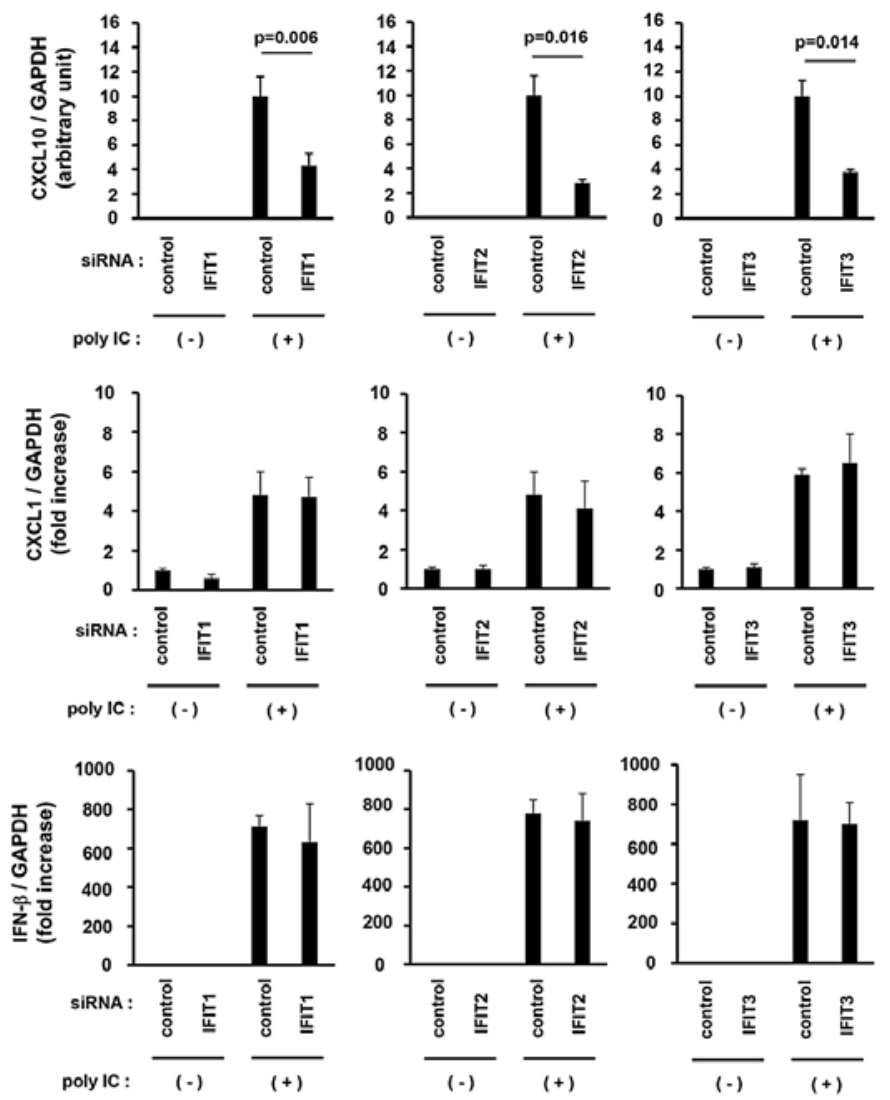

\section{B. ELISA}

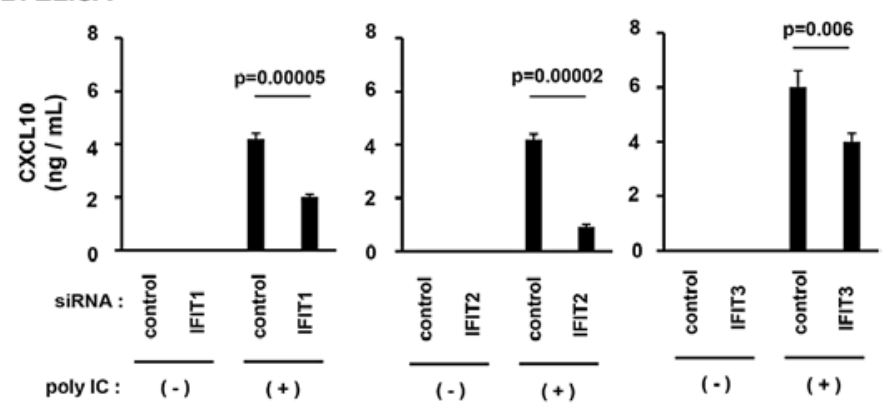

\section{C. western blotting}

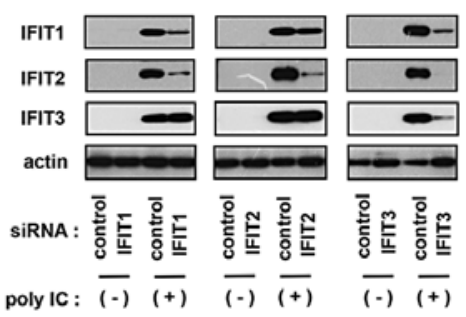




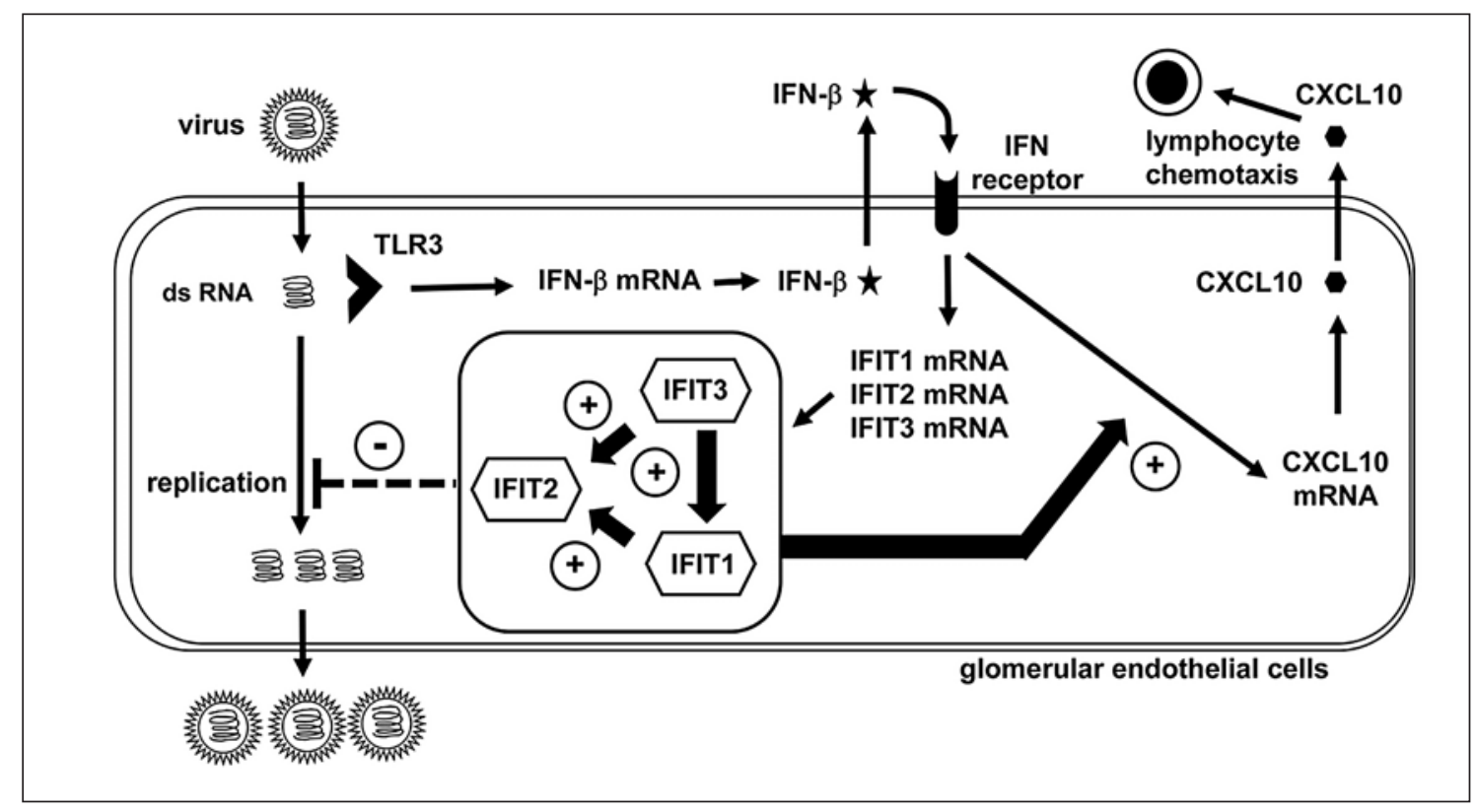

Fig. 5. Proposed role of IFIT1, IFIT2, and IFIT3 in the dsRNA-induced expression of CXCL10 in human GECs. Poly IC induces the expression of IFIT1, IFIT2, IFIT3, and CXCL10 via IFN- $\beta$. IFIT1, IFIT2, and IFIT3 proteins may inhibit viral replication (dashed line) and may positively regulate the expression of CXCL10 (thick arrow), which mediates lymphocyte chemotaxis. These reactions may be involved in antiviral and/or pseudoviral immunity. Poly IC, polyinosinic-polycytidylic acid; GECs, glomerular endothelial cells; IFIT1/2/3, tetratricopeptide repeats 1/2/3; CXCL, C-X-C motif chemokine ligand; IFN, interferon; dsRNA, double-stranded RNA; TLR, tolllike receptor.

CXCL10 mRNA reached a maximal level $16 \mathrm{~h}$ after the poly IC treatment and then plateaued, suggesting that CXCL10 mRNA was not degraded from 16 to $24 \mathrm{~h}$. However, marked expression of IFIT1 and IFIT2 proteins was also found $16 \mathrm{~h}$ after poly IC treatment. IFIT2 reportedly modulates the stability of cytokine mRNA by binding directly to RNA [25]. Although we do not have evidence that IFIT proteins can bind and stabilize the CXCL10 mRNA under the condition we tested, it is likely that IFIT proteins are involved in the stability of CXCL10 mRNA. Knockdown of IFIT1, IFIT2, or IFIT3 did not affect the poly IC-induced mRNA expression of CXCL1 and IFN- $\beta$, suggesting that IFIT proteins do not regulate IFN- $\beta /$ ISG signaling generically. Thus, IFIT1, IFIT2, and IFIT3 may increase antiviral and pseudoviral immune reactions in GECs, at least in part, by enhancing CXCL10 expression.

Since December 2019, the outbreak of a novel severe acute respiratory syndrome coronavirus 2 (SARS-CoV-2) has been a worldwide problem. In addition to the infection in lungs, SARS-CoV-2 can directly infect the kidney, and can cause acute kidney injury [36]. SARS-CoV-2 is an RNA virus, and there is a possibility that IFIT1, IFIT2, and IFIT3 expressed in GECs may play a protective role against the coronavirus infection. Although speculative, there is a possibility that understanding of the regulation of expression and function of IFIT proteins may contribute to the development of new therapeutic strategies for viral infection-related kidney diseases in the future.

There are some limitations in the present study. Although we have previously observed that TLR3 is involved in poly IC-induced signaling in the system we used [9], we cannot deny the possibility that poly IC has offtarget effects and is not acting through TLR3. We did not perform immunoprecipitation assays to detect proteinprotein and/or protein-RNA binding. The precise molecular mechanisms by which IFIT1, IFIT2, and IFIT3 positively regulate CXCL10 expression need to be investigated in future studies.

\section{Conclusion}

Poly IC induced the expression of IFIT1, IFIT2, IFIT3, and CXCL10 in an IFN- $\beta$-dependent manner in human GECs. IFIT1, IFIT2, and IFIT3 positively regulate the expression of CXCL10. IFIT1, IFIT2, and IFIT3 may func- 
tion, at least in part, in antiviral and pseudoviral innate immune reactions that enhance CXCL10 expression in GECs (Fig. 5).

\section{Statement of Ethics}

Human subjects and animals that require ethical approval were not involved in this study.

\section{Conflict of Interest Statement}

The authors have no conflicts of interest to declare.

\section{Funding Source}

This work was supported by Grant-in-Aid from the Japan Society of Promotion of Science (JSPS KAKENHI Grant No. 17K09681 to T.I.).

\section{Author Contributions}

Tadaatsu Imaizumi contributed to all of the procedure of the work. Shun Hashimoto contributed to cell culture, qRT-PCR, and Western blotting. Riko Sato and Hidenori Umetsu contributed to cell culture, qRT-PCR, and ELISA. Tomomi Aizawa and Shojiro Watanabe contributed to cell culture and designing the study. Shogo Kawaguchi, Tomoh Matsumiya, Kazuhiko Seya, and Jiangli Ding contributed to cell culture, Western blotting, and interpretation of data. Hiroshi Tanaka contributed to conception and designing of the work.

\section{References}

1 Lai AS, Lai KN. Viral nephropathy. Nat Clin Pract Nephrol. 2006;2(5):254-62.

2 Weidenbusch M, Kulkarni OP, Anders HJ. The innate immune system in human systemic lupus erythematosus. Clin Sci. 2017;131(8): 625-34.

3 Zindel J, Kubes P. DAMPs, PAMPs, and LAMPs in immunity and sterile inflammation. Annu Rev Pathol. 2020;15:493-518.

4 Miyake K, Shibata T, Ohto U, Shimizu T, Saitoh S-I, Fukui R, et al. Mechanisms controlling nucleic acid-sensing Toll-like receptors. Int Immunol. 2018;30(2):43-51.

5 Kariko K, Ni H, Capodici J, Lamphier M, Weissman D. mRNA is a endogenous ligand for Toll-like receptor 3. J Biol Chem. 2004; 279:12542-50.

6 Anders HJ, Banas B, Schlöndorff D. Signaling danger: toll-like receptors and their potential roles in kidney disease. J Am Soc Nephrol. 2004;15(4):854-67.

7 Tsuruga K, Aizawa T, Watanabe S, Tsugawa $\mathrm{K}$, Yoshida H, Imaizumi T, et al. Expressions of mRNA for innate immunity-associated functional molecules in urinary sediment in immunoglobulin A nephropathy. Nephrology. 2015;20(12):916-21.

8 Anders HJ. Pseudoviral immunity: a novel concept for lupus. Trends Mol Med. 2009; 15(12):553-61.

9 Liu Q, Imaizumi T, Kawaguchi S, Aizawa T, Matsumiya T, Watanabe S, et al. Toll-like receptor 3 signaling contributes to regional neutrophil recruitment in cultured human glomerular endothelial cells. Nephron. 2018; 139(4):349-58

10 Matsumoto M, Seya T. TLR3: interferon induction by double-stranded RNA including poly (I:C). Adv Drug Deliv Rev. 2008;60:80512.
11 Liu Q, Imaizumi T, Aizawa T, Hirono K, Kawaguchi S, Watanabe S, et al. Cytosolic sensors of viral RNA are involved in the production of interleukin- 6 via Toll-like receptor 3 signaling in human glomerular endothelial cells. Kidney Blood Press Res. 2019;44(1):6271.

12 Hirono K, Imaizumi T, Aizawa T, Watanabe S, Tsugawa K, Shiratori T, et al. Endothelial expression of fractalkine (CX3CL1) is induced by Toll-like receptor 3 signaling in cultured human glomerular endothelial cells. Modern Rheumatol. Forthcoming 2020.

13 Fensterl V, Sen GC. The ISG56/IFIT1 gene family. J Interferon Cytokine Res. 2011;31(1): 71-8.

14 Diamond MS, Farzan M. The broad-spectrum antiviral functions of IFIT and IFITM proteins. Nat Rev Immunol. 2013;13(1):4657.

15 Zhou X, Michal JJ, Zhang L, Ding B, Lunney $\mathrm{JK}$, Liu B, et al. Interferon induced IFIT family genes in host antiviral defense. Int J Biol Sci. 2013;9(2):200-8.

16 Habjan M, Hubel P, Lacerda L, Benda C, Holze C, Eberl CH, et al. Sequestration by IFIT1 impairs translation of $2^{\prime} \mathrm{O}$-unmethylated capped RNA. PLoS Pathog. 2013;9(10): e1003663.

17 Stawowczyk M, Van Scoy S, Kumar KP, Reich NC. The interferon stimulated gene 54 promotes apoptosis. J Biol Chem. 2011;286(9): 7257-66.

18 Hsu YL, Shi SF, Wu WL, Ho LJ, Lai JH. Protective roles of interferon-induced protein with tetratricopeptide repeats 3 (IFIT3) in Dengue virus infection of human lung epithelial cells. PLoS One. 2013;8(11):e79518.

19 Luster AD, Ravetch JV. Biochemical characterization of a gamma interferon-inducible cytokine (IP-10). J Exp Med. 1987;166(4): 1084-97.
20 Van Raemdonck K, Van den Steen PE, Liekens S, Van Damme J, Struyf S. CXCR3 ligands in disease and therapy. Cytokine Growth Factor Rev. 2015;26(3):311-27.

21 Elia G. Interferon- $\gamma$-inducible chemokines in systemic lupus erythematosus. Clin Ter. 2015; 166(1):e41-6.

22 Imaizumi T, Tanaka H, Matsumiya T, Yoshida H, Tanji K, Tsuruga K, et al. Retinoic acidinducible gene-I is induced by double-stranded RNA and regulates the expression of CC chemokine ligand (CCL) 5 in human mesangial cells. Nephrol Dial Transplant. 2010; 25(11):3534-9.

23 Pichilmair A, Lassnig C, Eberle CA, Gorna MW, Baumann CL, Burkard TR, et al. IFIT1 is an antiviral protein that recognizes $5^{\prime}$-triphosphate RNA. Nat Immunol. 2011;12:62430.

24 Kumar P, Sweeney TR, Skabkin MA, Skabkina OV, Hellen CU, Pestova TV. Inhibition of translation by IFIT family members is determined by their ability to interact selectively with the $5^{\prime}$-terminal regions of cap0-, cap1and 5'ppp-mRNAs. Nucleic Acids Res. 2014; 42(5):3228-45.

25 Yang Z, Liang H, Zhou Q, Li Y, Chen H, Ye W, et al. Crystal structure of ISG54 reveals a novel RNA binding structure and potential functional mechanisms. Cell Res. 2012;22(9): 1328-38.

26 Tian Q, Zhao H, Ling H, Sun L, Xiao C, Yin $\mathrm{G}$, et al. Poly(ADP-ribose) polymerase enhances infiltration of mononuclear cells in primary Sjogren's syndrome through interferon-induced protein with tetratricopeptide repeats 1-mediated up-regulation of CXCL10. Arthritis Rheumatol. 2020;72:1003-12. 
27 Imaizumi T, Numata A, Yano C, Yoshida H, Meng P, Hayakari R, et al. ISG54 and ISG56 are induced by TLR3 signaling in U373MG human astrocytoma cells: possible involvement in CXCL10 expression. Neurosci Res. 2014;84:34-42.

28 Imaizumi T, Yoshida H, Hayakari R, Xing F, Wang L, Matsumiya T, et al. Interferon-stimulated gene (ISG) 60 , as well as ISG56 and ISG54, positively regulates TLR3/IFN- $\beta /$ STAT1 axis in U373MG human astrocytoma cells. Neurosci Res. 2016;106:35-41.

29 Imaizumi T, Sassa N, Kawaguchi S, Matsumiya $\mathrm{T}$, Yoshida $\mathrm{H}$, Seya $\mathrm{K}$, et al. Interferonstimulated gene 60 (ISG60) constitutes a negative feedback loop in the downstream of TLR3 signaling in hCMEC/D3 cells. J Neuroimmunol. 2018;324:16-21.
30 Li Y, Li C, Xue P, Zhong B, Mao AP, Ran Y, et al. ISG56 is a negative-feedback regulator of virus-triggered signaling and cellular antiviral response. Proc Natl Acad Sci U S A. 2009; 106(19):7945-50.

31 Conti F, Spinelli FR, Truglia S, Miranda F, Alessandri C, Ceccarelli F, et al. Kidney expression of Toll like receptors in lupus nephritis: quantification and clinicopathological correlations. Mediators Inflamm. 2016; 2016:7697592.

32 Mavragani CP, Sagalovskiy I, Guo Q, Nezos A, Kapsogeorgou EK, Lu P, et al. Expression of long interspersed nuclear element 1 retroelements and induction of type I interferon in patients with systemic autoimmune disease. Arthritis Rheumatol. 2016;68(11):2686-96.
33 Maie MA, Khalil RE, Habib HM. Urinary CXCL10: a marker for nephritis in lupus patients. Reumatismo. 2014;65:292-7.

34 Fleith RC, Mears HV, Leong XY, Sanford TJ, Emmott E, Graham SC, et al. IFIT3 and IFIT2/3 promote IFIT1-mediated translation inhibition by enhancing binding to non-self RNA. Nucleic Acids Res. 2018;46(10):526985.

35 Johnson B, Vanblargan LA, Xu W, White JP, Shan C, Shi PY, et al. Human IFIT3 modulates IFIT1 RNA binding specificity and protein stability. Immunity. 2018;48(3):487-99.e5.

36 Martinez-Rojas MA, Vega-Vega O, bobadilla NA. Is the kidney a target of SARS-CoV-2? Am J physiol Renal Physiol. 2020;318(6): F1454-62. 